\title{
PCI Matrix Based Image Reconstruction from Compressively Sensed Data
}

\author{
B. Mounika, B. Sridhar, S.V.S.Prasad
}

\begin{abstract}
This paper propounds a joint system wherever in raise to a higher position-based, distinguishable, picture coordinated wavelets are measurable from squeezeingly seen pictures and are utilized for the remaking of indistinguishable. Coordinated swell is just outlined if full picture is advertised. Conjointly contrasted and the quality wavelets as scarifying bases, coordinated swell may surrender higher remaking closes in compressive detecting (CS) application. Since in metallic component application, we have squeezeingly seen pictures as opposed to full pictures, existing methods for arranging coordinated swells can't be utilized. In this way, we tend to propose a joint structure that evaluations coordinated wavelets from squeezeingly saw pictures and conjointly remakes full pictures.

3 Indispensable commitments. Initial, a lifting-based, picture coordinated severable swell is implied from compressively seen pictures and is furthermore wont to remake indistinguishable. Second, a simple detecting lattice is utilized to test data at sub-NY Quist rate such detecting and remaking time is decreased essentially. Third, a substitution staggered L-Pyramid swell decay procedure is accommodated severable swell execution on pictures those outcomes in enhanced remaking execution. Contrasted and the CS-based recreation misuse typical swells with Gaussian detecting network and with existing swell decay system, the arranged approach gives snappier and higher picture reproduction in metallic component application.

Index Terms-Compressive sensing, matched wavelet, lifting, wavelet decomposition.
\end{abstract}

\section{INTRODUCTION}

Established flag securing method in flag process branch includes detecting the total flag at or over the NY Quist rate. By and large, this flag is revamped to a site wherever it's compressible. Only the absolute most imperative coefficients of revamped flag having enough amount of vitality are hang on and transmitted to the collector in conjunction with the locale statistics of the transmitted palimpsest the Associate Editor coordinative the survey technique was employee. Yuen M. Lu. (Comparing creator: Naushad Ansari.) The transmitted flag is decoded at the recipient to recoup the underlying sign. In this manner, this technique includes detecting the entire flag, however a large portion of the examples inside the revised area are to be disposed of. In [1] and [2], scientists arranged packed/compressive detecting (CS) approach that blends detecting and pressure in one phase, wherever instead of inspecting a proof example astute over NY Quist rate, flag projections are caught by means of a measure premise. These examples are just a couple when contrasted with those examined at Nytquist rate. On the off chance that the flag is

Revised Manuscript Received on July 18, 2019.

B. Mounika, PG Student, Department of ECE, MLR Institute of Technology, Hyderabad, India

B. Sridhar, Professor, Department of ECE, MLR Institute of Technology, Hyderabad, India

S.V.S.Prasad, Professor, Department of ECE, MLR Institute of Technology, Hyderabad, India appropriated in some rebuild area and also if the measure premise is ambiguous with the scarifying premise, at that point the entire flag will be remade with a legitimate probability from a horrendously couple of projections of the flag. Amid this specific situation, wavelets are widely utilized as scarifying premise in packed detecting drawback [2]. One among the favourable position with wavelets is that there's no unmistakable premise as opposed to Fourier redesign. One may choose the arrangement of premise depending upon the kind of use. Since the swell premises aren't unmistakable, it's higher to style swell that are coordinated to a given flag in an exceedingly particular application. The planned swell premises are known as flag coordinated wavelets [3] - [5]. Expected with the over dialog, this paper proposes to style coordinated wavelets for compressive detecting application. As opposed to past deals with coordinated wavelets wherever wavelets are planned

\section{MATHEMATICAL MODELLING OF IMAGE RECONSTRUCTION}

A picture might be a flag conveying data a couple of protest that isn't specifically noticeable. Ordinarily the information comprises of a corrupted delineation of the underlying article and one wills generally separate2sources of debasement: technique the method\} of picture arrangement and furthermore the procedure of picture recording. The corruption on account of the technique for picture arrangement is normally signified by obscuring and might be a style of band constraining of the thing. Inside the instance of elevated pictures, incidentally, the obscuring is because of relative movement between the camera and furthermore the ground, to distortions of the optical parts of the camera and, at last, to environmentally disturbance. The debasement presented by the account technique is commonly indicated by commotion and is because of measure mistakes, researching blunders, and so on. Obscuring might be a settled technique and, much of the time, one fuses an adequately adjust numerical model for its portrayal.

$$
\operatorname{PSNR}(\text { in } d B)=10 \log _{10}\left(\frac{(\max (I))^{2}}{\sum_{i=0}^{m-1} \sum_{j=0}^{n-1}|I(i, j)-\hat{I}(i, j)|^{2}}\right)
$$

\section{THE LOSS OF DATA THANKS TO ASSOCIATE DEGREE OPTICAL SYSTEM}

Condition 1.1 is regularly seen in intentional investigation as an administrator between capacities territories. we tend to consider that the articles zone $\mathrm{O}$ and furthermore the photos

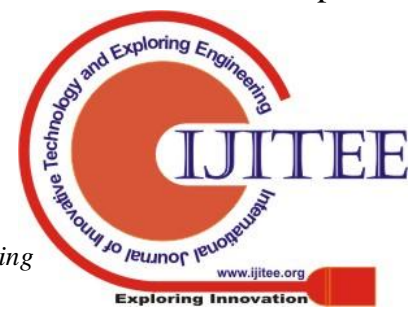


zone I square measure David Hilbert regions, The imaging framework will transmit finish data concerning the thing or not. From a scientific motivation behind read, this fact is preoccupied by recommends that of the attributes of the administrator $\mathrm{H}$. It speaks to the transmission of finish information if and on condition that it's invertible, i.e. At the point when the essential condition bombs, at that point there exist at least 2 unmistakable items, say $\mathrm{f} 1$ and $\mathrm{f} 2$, indicated $\mathrm{Hf} 1=\mathrm{g}$ and $\mathrm{Hf} 2=\mathrm{g}$. Since the administrator $\mathrm{H}$ is direct, we get $\mathrm{H}(\mathrm{f} 1-\mathrm{f} 2)=0$ and in this manner $\mathrm{f}=\mathrm{fl}-\mathrm{f} 2$ is a nonminor arrangement of the homogeneous condition $\mathrm{Hf}=0$. On the other hand, if this condition consolidates a non-minor goals $f$ and $\mathrm{f} 1$ could be a goal of condition a couple of. 1 ,

$$
\text { i.e. } \quad \mathrm{Hf} 1=\mathrm{g} \text {, then } \mathrm{f} 2=\mathrm{f} 1+\mathrm{f}
$$

Is additionally an answer of a similar equation as a result of

$$
\mathrm{Hf} 2=\mathrm{Hf} 1+\mathrm{Hf}=\mathrm{Hf} 1=\mathrm{g} \text {. }
$$

We tend to see that the appropriate response of the condition $\mathrm{Hf}=\mathrm{g}$ is elite if and on condition that the condition $\mathrm{Hf}=$ zero has exclusively the appropriate response $\mathrm{f}=$ zero. Once there exist non-trifling arrangements, these speak to a straight s subspace numerical region topological space\} ker $\mathrm{H}$ known as the scientific space of the administrator $\mathrm{H}$ or also the space of the undetectable articles. To determine the presence of imperceptible articles it's helpful to scribble down the condition $\mathrm{Hf}=$ zero regarding Fourier changes.

\section{POSTULATION OF DIGITAL IMAGE PROCESSING}

By and elephantine an flourish portrayal is depicted in smallest components that zone unit thought of as jiffy parts of a photo or conjointly denominated as pix. A pel might be a blend of eight bits fabricate out of each vital most vital|most important\} bits and also as minimum huge bits. Here a vital reason for existing is that the premier essential is in danger of pandemonium or the supplemental heterogeneity in shine, metamorphose, goals it'll effect essentially on slightest critical bits because of its confessment demeanour. The different bits territory unit depicted zone unit appearance as appeared inside the figure 3.2. These bits in an exceedingly pel territory unit sorted out inside the plummet deportment wherever utmostone of the eight gobbets power is emerged in bulk indispensable fragments everything being equal. Cybernated picture powers zone unit bargains on the furtherance consequence of those fragments in an exceedingly rectify approach in this manner on envision in an exceedinglycorrectthanks to human tangible scafolding (HVS).

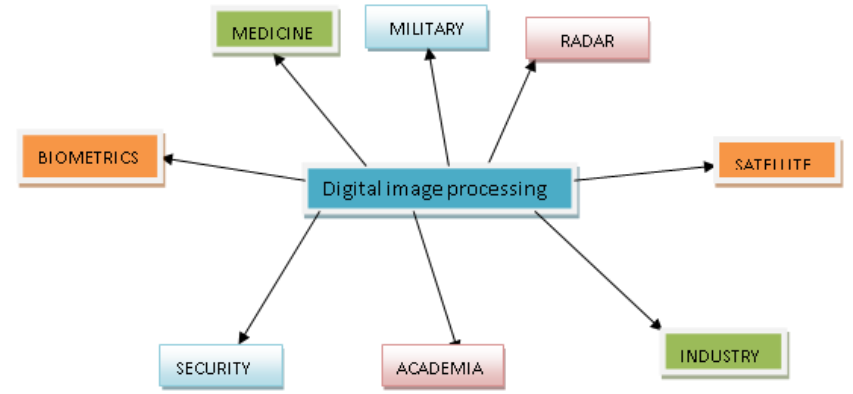

FIGURE 1:Digital image processing applications

\section{Literature Survey:}

Anubha Gupta This paper proposes a substitution system of assessing each symmetrical briefly bolstered besides as semi-symmetrical boundlessly/minimalistically upheld riffle from a given flag. The strategy is predicated on expanding projection of the given flag onto serials calling numerical space. This winds up in decrease of vitality of flag inside the riffle numerical space. The idea acclimated assess examination riffle channel is similar to a honing channel used in picture sweetening. Initial, a substitution procedure is arranged that aides inside the style of 2-band FIR symmetrical great recreation channel bank from a given flag.

This wind up in the arranging of biorthogonal compactly upheld riffle. It's conjointly demonstrated that a riffle with wanted help besides as wanted scope of vanishing minutes is outlined with the arranged strategy. Next, a way is wanted to style semi-symmetrical wavelets that region unit ordinarily limitlessly bolstered wavelets. Here, comparing to FIR examination channels, the following union channels region unit IIR channels that fulfilthe property of good remaking.

In this paper, a substitution system of evaluating coordinated riffle has been arranged. The key arrangement exists in the estimation of research riffle channel from a given flag. The idea to assess this channel is comparable to a honing channel used in picture sweetening. Upheld this approach, first investigation riffle channel is measurable. When investigation riffle channel is measurable, the issue is an approach to style examination scaling channel. There's no method to search out scaling channel unambiguously from riffle channel.

MRA is elite comparable to scaling perform anyway proportionate to riffle perform we will have a few scaling capacities and in this manner MRA. Along these lines, while evaluating examination riffle channel, the issue is then seen from the flag procedure side and thusly issue of good remaking is talked concerning. Here, it's to push that we will have a few exchange styles, as biorthogonal, semisymmetrical and symmetrical riffle style. To start with, we tend to focus on PR biorthogonal FIR channel bank that winds up in style of biorthogonal compactly upheld coordinated riffle. Next, we tend to expected riffle scientific space to be symmetrical to scaling numerical space and along these lines specified the arranging of semisymmetrical riffle that zone unit normally boundlessly bolstered wavelets, while style of symmetrical wavelets was offered by the creators in [11]. The arranged method is connected on 2 world clasps: one music and one discourse cut. Here, it's resolved that the following scaling capacities show up as though splines, Coif let or Daubechies scaling perform or takes another shape. Utilization of thought of coordinated riffle is appeared inside the setting of flag pressure. it's resolved that coordinated riffle said here offers higher outcomes for pressure when contrasted with plain wavelets biorthogonal $9=7$ and $5=3$ : the strategy is more reached out to make a coordinated parallel tree-like structure prompting channel keep money with pulverization quantitative connection of forces of 2 .

Blue Eyes Intelligence Engineering 


\section{SQUEEZEING COMPREHEND OF PORTRAITS MANOEURVE PROPOUND SUSPECTING MATRIX}

\section{PROPOSED METHODE:}

In this segment, beginning we tend to build up the necessity for an unmistakable, anyway ordinary, detecting network. Next, we tend to examine the arranged network. What's more, later, we tend to blessing results to call attention to the examination of your chance quality and recreation execution with the arranged grid in cesium based for the most part picture remaking.

\section{Context}

In this day and age, size of pictures are more monstrous and $\mathrm{N}$ normally ways to deal with army tests. This monstrous size imaging postures challenges for CS-based picture remaking. Beginning test is that the immense size of estimating lattice that stances issues with capacity and calculation. Elective issues grasp style of imaging framework with bigger house data measure item (SBP) and extreme institutionalization needs [31]. In a preliminary to beat the over difficulties, single pel camera equipment configuration arranged.

Appeal re-establish a standard Polaroid outlines, catches the scalar item interpolated pageant underneath read \& estimating premise. In this way, the camera catches one pel during a period direct mix pel test of the picture. Following Technique is repetitive $\mathrm{M}$ scope allotment amidst $\mathrm{M} * \mathrm{~N}$. This are alluded to as the squeezing estimations furthermore ensue disseminate recipient wherever impregnated measured picture remade by utilizing possibility of CSbased reproduction. Considering extra propaganda forth secluded pelcamera, per user authorized take a seat with [18]. With the over clarified outline, one pel camera replaces the check boson indicator exhibit of a customary camera by one gauge boson finder; along these lines, diminishing the scale, cost, and nature of the imaging plan.

\section{Planned manoeuvre of fragmentary Authoritative} Congruence Appprehend Matrix

Propound to utilize detecting framework comprehension, that the least complex detecting network arranged up to this point and "really" faculties the picture at by catching barely scope of pels while not detecting information concerning every pixel [32]. This can be clarified as beneath.

Consider a photo $\mathrm{X}$ of measurement $\mathrm{m} \times \mathrm{n}$. as opposed to examining the picture exploitation the detecting component cluster of the standard Polaroid, we tend to catch $\mathrm{M}$ tests of the picture exploitation the arranged estimating framework estimating framework $\mathrm{p}$ has the passages demonstrated as follows:

Where, $\subset$ indicated $\|=\mathrm{M}$ and |.|. This detecting lattice is thought as fractional authoritative personality grid of it comprises incompletely picked and permuted columns of the scalar network.

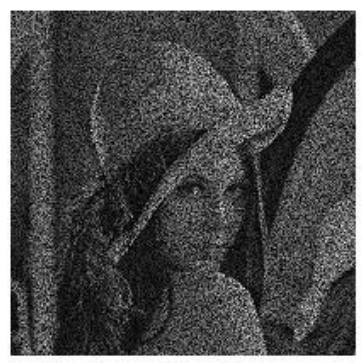

(a)

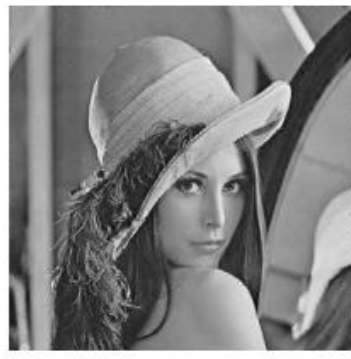

(b)
FIGURE 2: (a) Image (with dimension $512 \times 512$ ) captured victimization PCI sensing matrix with five hundredth sampling magnitude relation and with zeros stuffed at positions not sampled (b) Image reconstructed from sub-sampled image victimization ' $d b 4$ ' .

Planned L-Pyramid Ripple Decomposition Techniques For Pictures

As communicated before, we tend to need to assess coordinated swell for each the line and segment headings. One simpler method might concoct coordinated swell on line or segment defrauded picture and utilize indistinguishable swell, later, on each the segments and columns as a severable swell. Rather, we have a propensity to propose to style coordinated swell separately for the line and segment bearings exploitation the ensuing 2 filtering designs:

- arrangement filtering design: The picture is examined with regards to the checking design appeared in Fig. 8, whereby lines or sections are stacked one when the inverse to Fig.8.

Development examining design $\mathrm{Rm}$ indicates the mth line and $\mathrm{Cn}$ signifies the ordinal segment. (a) Column savvy arrangement examining.

\section{(b) Row shrewd arrangement filtering.}

Fig. 9. twisted checking design $\mathrm{Rm}$ means the mth line and $\mathrm{Cn}$ indicates the ordinal section. (a) Column insightful twisted checking. (b) Row insightful twisted examining. secure 1-D motion for each the headings.

Be that as it may, this may cause partition inside the 1-D motion at the changes once one segment closes and another begins and in like manner, for the lines.

- twisted filtering design: in order to keep away from this partition, Associate in Nursing substitute means is to check every even line or sections inside the switch course as appeared in Fig. 9.

Since twisted checking design is great to unanticipated advances at the line or segment endings, we tend to utilize everything told our tests.

\section{B. arranged Methodology of Matched swell style}

With the twisted filtering said over, we tend to change over a given picture into 2 1-D signals: one with segment astute examining and another with push shrewd checking. From now on, inside the blessing Section, we tend to blessing coordinated swell style philosophy exploitation compressively recognized 1-D flag. we will utilize these styles inside the following Section to see the execution over pictures as severable swell changes. 


\section{RESULTS}

In this paper, we have arranged a joint structure whereby picture coordinated wavelets are composed from compressively apparent pictures and later, utilized for recreation or recuperation of the total picture. we have conjointly intended to utilize a halfway authoritative character detecting framework for CS-based reproduction of pictures that plays out a great deal of speedier contrasted with the present Gaussian or Bernoulli grids and in this way, is fitted to time-bound day and age recreation based for the most part applications.

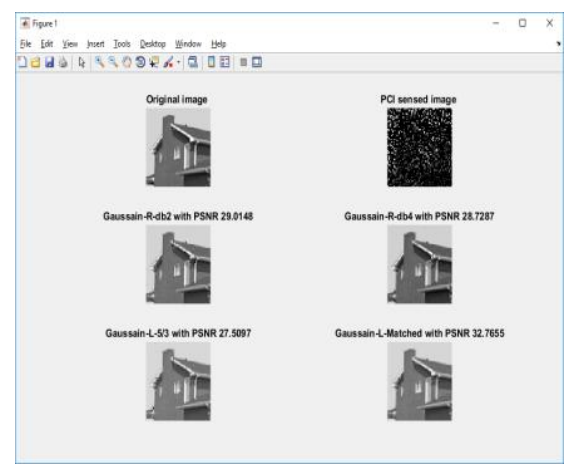

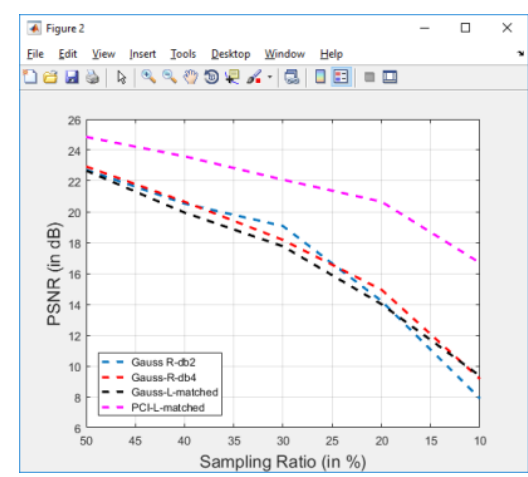

FIGURE 3: Sampling Ratio vs. PSNR for existing and proposed work

FIGURE 4: a) Original image b) PCI sensed image c)Gaussian R-db2 with PSNR 29.0148 d)Gaussian R-db4 with PSNR 28.7247 e)Gaussian L-5/3 with PSNR 27.5097 f) c)Gaussian L-matched with PSNR 32.7655.

TABLE : Reconstruction accuracy on CS-based image reconstruction with standard wavelets and with imagematched wavelets designed from compressively sensed images with 5/3 length filters. PCI sensing matrix and the proposed L-pyramid wavelet decomposition have been used to generate these results

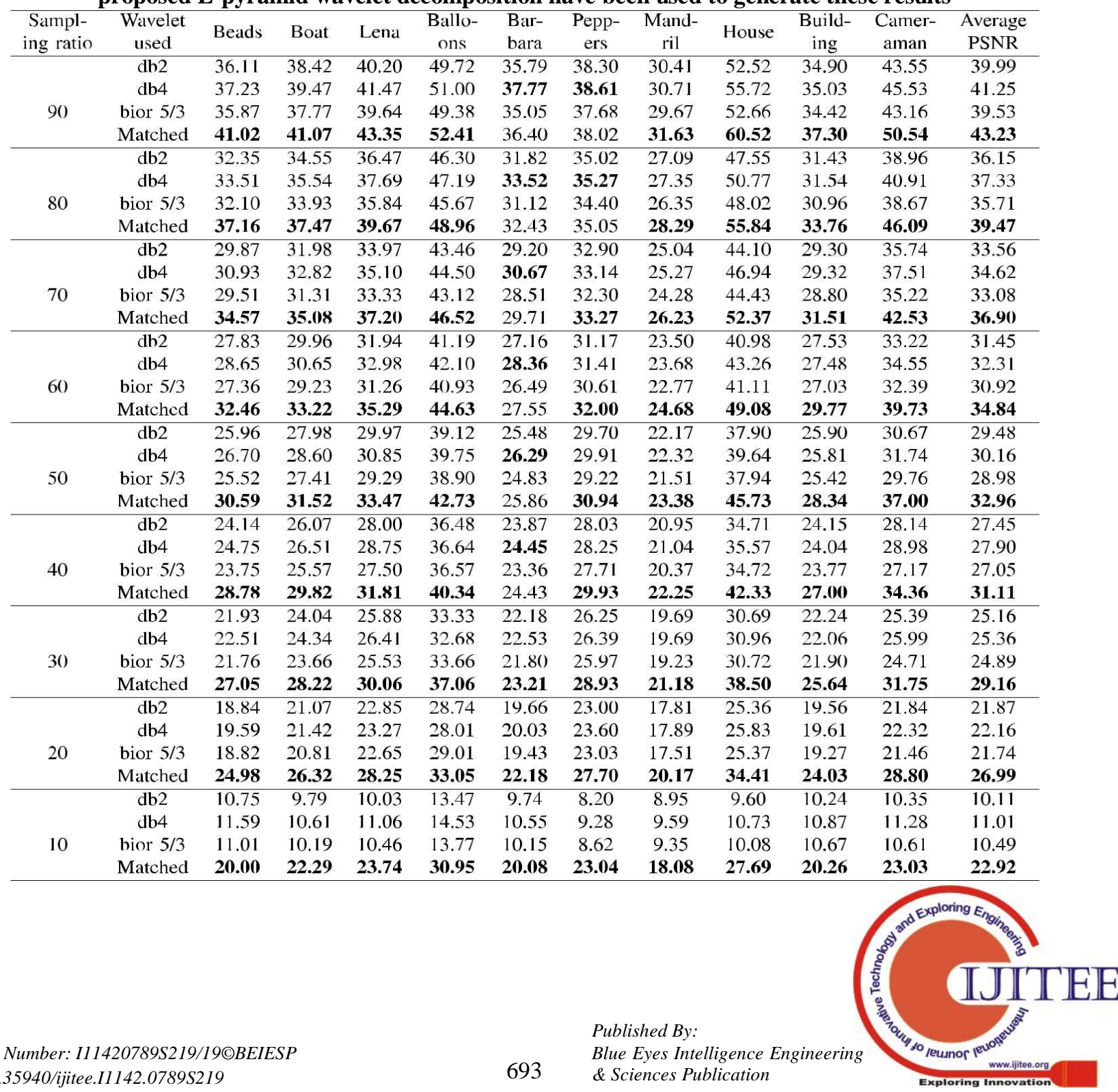




\section{CONCLUSION}

Thought here's a little corruption in execution with the arranged detecting network anyway that is essentially covered up by the coordinated riffle Style. We have conjointly given a substitution staggered L-Pyramid riffle decay strategy that works undeniably with proficiency contrasted with the quality riffle decomposition methodology. Generally speaking, the arranged work with very surprising detecting network, new riffle decomposition strategy, and picture coordinated wavelets offer obviously better remaking outcomes with simple equipment execution in CS-based picture recreation contrasted with the present technique.

\section{REFERENCES}

1. E. J. Candès, J. Romberg, and T. Tao, "Robust uncertainty principles: actual signal reconstruction from extremely incomplete frequency data," IEEE Trans. Inf. Theory, vol. 52, no. 2, pp. 489-509, Feb. 2006.

2. D. L. Donoho, "Compressed sensing," IEEE Trans. Inf. Theory, vol. 52, no. 4, pp. 1289-1306, Apr. 2006.

3. A. Gupta, S. D. Joshi, and S. Prasad, "A new methodology of estimating riffle with desired options from a given signal," Signal method., vol. 85, no. 1, pp. 147-161, Jan. 2005.

4. A. Gupta, S. D. Joshi, and S. Prasad, "A new approach for estimation of statistically matched riffle," IEEE Trans. Signal method., vol. 53, no. 5, pp. 1778-1793, May 2005.

5. N. Ansari and A. Gupta, "Signal-matched riffle style via lifting mistreatment optimisation techniques," in Proc. IEEE Int. Conf. Digit. Signal method. (DSP), Jul. 2015, pp. 863867.

6. J. O. Chapa and R. M. Rao, "Algorithms for planning wavelets to match a mere signal," IEEE Trans. Signal method., vol. 48, no. 12, pp. 3395-3406, Dec. 2000.

7. R. L. Claypoole, R. G. Baraniuk, and R. D. Nowak, "Adaptive riffle transforms via lifting," in Proc. IEEE Int. Conf. Acoust., Speech Signal method., vol. 3. May 1998, pp. 1513-1516.

8. G. Piella, B. Pesquet-Popescu, and H. J. A. M. Heijmans, "Gradientdriven update lifting for accommodative wavelets," Signal method., Image Commun., vol. 20, nos. 9-10, pp. 813831, Oct./Nov. 2005.

9. H. J. A. M. Heijmans, B. Pesquet-Popescu, and G. Piella, "Building nonredundant accommodative wavelets by update lifting," CWI, Amsterdam, European nation, Res. Rep. PNAR0212, 2002.

10. W. Sweldens, "The lifting scheme: A custom-design construction of biorthogonal wavelets," Appl. Comput. Harmon. Anal., vol. 3, no. 2, pp. 186-200, Apr. 1996.

11. W. Dong, G. Shi, and J. Xu, "Signal-adapted directional lifting theme for compression," in Proc. IEEE Int. Symp. Circuits Syst., May 2008, pp. 1392-1395.

12. G. Quellec, M. Lamard, G. Cazuguel, B. Cochener, and C. Roux, "Adaptive nonseparable riffle rework via lifting and its application to content-based image retrieval," IEEE Trans. Image method., vol. 19, no. 1, pp. 25-35, Jan. 2010. 